\title{
The role of the Instrument Control Unit within the ARIEL Payload and its current design
}

\author{
Luca Naponiello, Vladimiro Noce, Mauro Focardi, Anna Maria Di
} Giorgio, Giampaolo Preti, Andrea Lorenzani, Andrea Tozzi, Ciro Del Vecchio, Maria Farina, Emanuele Galli, Gianluca Morgante, Antonio Scippa, Gabriele Redigonda, Giovanni Giusi, Jerome Amiaux, Christophe Cara, Michel Berthe, Roland Ottensamer, Paul Eccleston, Andrew Caldwell, Georgia Bishop, Lucile Desjonqueres, Rachel Drummond, Daniele Brienza, Emanuele Pace

\section{Published version information:}

Citation: L Naponiello et al. The role of the Instrument Control Unit within the ARIEL Payload and its current design. In Space Telescopes and Instrumentation 2020: Optical, Infrared, and Millimeter Wave, Online, 14-18 Dec 2020, (2020): 48

DOI: $\underline{10.1117 / 12.2560504}$

Copyright (2020) Society of Photo-Optical Instrumentation Engineers (SPIE). One print or electronic copy may be made for personal use only. Systematic reproduction and distribution, duplication of any material in this publication for a fee or for commercial purposes, and modification of the contents of the publication are prohibited.

This version is made available in accordance with publisher policies. Please cite only the published version using the reference above. This is the citation assigned by the publisher at the time of issuing the AAM/APV. Please check the publisher's website for any updates.

This item was retrieved from ePubs, the Open Access archive of the Science and Technology Facilities Council, UK. Please contact epublications@stfc.ac.uk or go to http://epubs.stfc.ac.uk/ for further information and policies. 


\section{The role of the instrument control unit within the ARIEL Payload and its current design}

Naponiello, Luca, Noce, Vladimiro, Focardi, Mauro, Di Giorgio, Anna Maria, Preti, Giampaolo, et al.

Luca Naponiello, Vladimiro Noce, Mauro Focardi, Anna Maria Di Giorgio, Giampaolo Preti, Andrea Lorenzani, Andrea Tozzi, Ciro Del Vecchio, Maria Farina, Emanuele Galli, Gianluca Morgante, Antonio Scippa, Gabriele Redigonda, Giovanni Giusi, Jerome Amiaux, Christophe Cara, Michel Berthe, Roland Ottensamer, Paul Eccleston, Andrew Caldwell, Georgia Bishop, Lucile Desjonqueres, Rachel Drummond, Daniele Brienza, Emanuele Pace, "The role of the instrument control unit within the ARIEL Payload and its current design," Proc. SPIE 11443, Space Telescopes and Instrumentation 2020: Optical, Infrared, and Millimeter Wave, 114434P (13 December 2020); doi: $10.1117 / 12.2560504$ 


\title{
The role of the Instrument Control Unit within the ARIEL Payload and its current design
}

Luca Naponiello $^{\mathrm{a}}$, Vladimiro Noce ${ }^{\mathrm{a}}$, Mauro Focardi ${ }^{\mathrm{b}}$, Anna Maria Di Giorgio ${ }^{\mathrm{c}}$, Giampaolo Preti $^{\mathrm{a}}$, Andrea Lorenzani ${ }^{\mathrm{b}}$, Andrea Tozzi ${ }^{\mathrm{b}}$, Ciro Del Vecchio ${ }^{\mathrm{b}}$, Maria Farina ${ }^{\mathrm{c}}$, Emanuele Galli ${ }^{\mathrm{c}}$, Gianluca Morgante ${ }^{\mathrm{d}}$, Antonio Scippa ${ }^{\mathrm{e}}$, Gabriele Redigonda ${ }^{\mathrm{a}}$, Giovanni Giusic ${ }^{\mathrm{c}}$, Jerome Amiaux $^{f}$, Christophe Cara ${ }^{\mathrm{f}}$, Michel Berthe ${ }^{\mathrm{f}}$, Roland Ottensamer ${ }^{\mathrm{g}}$, Paul Eccleston ${ }^{\mathrm{h}}$, Andrew Caldwell $^{\mathrm{h}}$, Georgia Bishop ${ }^{\mathrm{h}}$, Lucile Desjonqueres ${ }^{\mathrm{h}}$, Rachel Drummond ${ }^{\mathrm{h}}$, Daniele Brienza ${ }^{\mathrm{c}}$, and Emanuele Pace ${ }^{\mathrm{a}}$

${ }^{a}$ Università degli Studi di Firenze, Dipartimento di Fisica e Astronomia, 50125 Firenze, Italy bINAF, Osservatorio Astrofisico di Arcetri, Largo E. Fermi 5, 50125 Firenze, Italy ${ }^{\mathrm{c}}$ IAPS, Istituto di Astrofisica e Planetologia Spaziali, Via del Fosso del Cavaliere 100, 00133 Roma, Italy

dINAF - Istituto di Astrofisica Spaziale e Fisica Cosmica - 40129 Bologna, Italy eUniversità degli Studi di Firenze, Dipartimento di Ingegneria Industriale, 50139 Firenze, Italy

${ }^{\mathrm{f}} \mathrm{CEA}$ - Irfu, Département d'Astrophysique - 91191 Gif-sur-Yvette, France gUniversität Wien - Institut für Astrophysik - 1180 Wien, Austria

${ }^{\mathrm{h}} \mathrm{RAL}$ Space - Rutherford Appleton Laboratory - OX11 0QX Harwell Oxford, UK

\begin{abstract}
ARIEL, the Atmospheric Remote-sensing Infrared Exoplanet Large-survey mission ${ }^{1-3}$ was selected in early 2018 by the European Space Agency (ESA) as the fourth medium-class mission (M4) launch opportunity of the Cosmic Vision Program, with an expected launch in late 2028. It is the first mission dedicated to the analysis of the chemical composition and thermal structures of up to a thousand transiting exoplanets atmospheres, which will expand planetary science far beyond the limits of our current knowledge.
\end{abstract}

The ARIEL Payload $(\mathrm{P} / \mathrm{L})^{4-6}$ is designed to carry out transit spectroscopy during both primary and secondary planetary eclipses, which in the end will form a wide picture on the nature of exoplanets atmospheres and their interiors, by determining the key factors that influence the formation and evolution of these planetary systems. $^{7,8}$ ARIEL will not only observe warm and hot transiting gas giants, but also super-Earths and Neptunes around a large range of host star types, however it will target planets hotter than $600^{\circ} \mathrm{K}$ to take advantage of their well-mixed atmospheres. The mission will exploit the spectral range between 1.10 and $7.80 \mu \mathrm{m}$, and broadband photometry in the optical $(0.50-0.80 \mu \mathrm{m})$ and Near IR $(0.80-1.10 \mu \mathrm{m})$.

The Fine Guidance System (FGS) is one of the instruments of the Payload and it contains three photometric channels (two of which are used for guiding as well as science) between 0.5-1.1 $\mu \mathrm{m}$ and a low resolution NIR spectrometer for the 1.1-1.95 $\mu \mathrm{m}$ range. An IR Spectrometer (AIRS) ${ }^{9}$ is also foreseen along with the FGS, as it will provide low resolution spectroscopy in two IR channels: Channel 0 (CH0) for the 1.95 to $3.90 \mu \mathrm{m}$ band and Channel 1 (CH1) for the 3.90 to $7.80 \mu \mathrm{m}$ range. At last, an Active Cooler System (ACS) that includes a Ne JouleThomson cooler is adopted to give active cooling capability to the AIRS detectors, which will work at cryogenic temperatures. AIRS is meant to be located at the intermediate focal plane of the telescope and common optical system and it will host two HgCdTe-based hybrid IR detectors and two cold front-end electronics (CFEE) for

Further author information: (Send correspondence to Mauro Focardi)

Mauro Focardi: E-mail: mauro.focardi@inaf.it, Telephone: +39-055-2752-260

Vladimiro Noce: E-mail: vladimiro.noce@inaf.it

Space Telescopes and Instrumentation 2020: Optical, Infrared, and Millimeter Wave, edited by Makenzie Lystrup, Marshall D. Perrin, Proc. of SPIE Vol. 11443, 114434P · @ 2020 SPIE CCC code: $0277-786 X / 20 / \$ 21 \cdot$ doi: $10.1117 / 12.2560504$ 
detectors control and readout. Each CFEE is driven by a Detector Control Unit (DCU), which is part of AIRS but is hosted within and managed by the Instrument Control Unit (ICU) ${ }^{10}$. ICU is a warm unit that is located inside the spacecraft Service Module (SVM) and it is based on a cold redundant configuration with the Power Supply Unit (PSU) and the Commanding and Data Processing Unit (CDPU) boards; both DCUs are instead cross-strapped and can be managed by the nominal or the redundant (PSU+CDPU) chain. ICU is in charge of AIRS management, collecting scientific and housekeeping (HK) telemetries from the spectrometer and HK from the telescope (temperatures readings), the P/L Optical Bench (OB) and other Subsystems (SS), because of a warm slave unit (TCU, Telescope Control Unit) interfaced to the ICU. After being collected, Science and HK telemetries are then forwarded to the S/C for temporary storage, before finally sending them to Ground. In this short paper we describe the status of the ICU design at the end of B1 Phase, just after the Mission Adoption Review (MAR) by ESA, and explain some still open architectural choices to be addressed and finalised once the ICU industrial Prime contractor is selected.

Keywords: Exoplanets Atmospheres, Transit Spectroscopy, Infrared Spectrometer, Payload Electronics, Instrument Control Unit

\section{INTRODUCTION}

The current architecture of the Payload and the ARIEL Mission Consortium (AMC) structure are depicted in the block diagram of Figure 1. The diagram shows the ICU, hosted inside the Service Module of the satellite, and some control electronics units: the FCU ${ }^{11}$ (FGS Control Unit or Fine Guidance System), the TCU (Telescope Control Unit) ${ }^{12}$ and the CCE (Cryocooler Control Electronics) ${ }^{13}$ for the active cryocooler (ACS) management. The Instrument Control Unit is the electronic unit in charge of managing the TCU, the whole AIRS Spectrometer and processing its collected scientific data, prior to sending it to the on-board data handling system of the Spacecraft (S/C).

The ICU is a subsystem conceived as an assembly with a mechanical enclosure provided of a back panel hosting the internal connectors to plug and interface the electronics boards, and several externally mounted connectors for electrically interfacing the outside world (e.g. Payload and Spacecraft). Its architecture relies on a cold redundant and partially cross-strapped configuration which host five sub-units with the following operational configuration:

- 1 PSU - Power Supply Unit (Nominal and Redundant);

- 1 CDPU - Commanding and Data Processing Unit (Nominal and Redundant);

- 2 DCU - Detector Control Unit (only Nominal) for the AIRS CH0 and CH1 channels;

- 1 BP - Backplane;

The ICU is also linked, by a serial cross-strapped interface (I/F), to the external Telescope Control Unit box ( $\mathrm{N}$ and $\mathrm{R}$ chains, in a cold redundant configuration), which manages the Thermal Control stabilisation System (TCS for operational heaters and thermistors) of the M1 and M2 mirrors, the M2 mechanism (M2M) and the onboard calibration source (OBCS). The ICU processor, the only chip running a high-level SW (Application SW), will control both DCUs and TCU as well, as the latter will implement only a slave FPGA (Field Programmable Gate Array), in order to make P/L operations and control more efficient and overall simple.

Italy (INAF, for the Italian Space Agency - ASI and its Prime/Industrial Contractor, to be selected in 2021) is responsible for the ICU design (enclosure included), the Power Supply Unit, the back panel and the Commanding and Data Processing Unit (including HW drivers, boot SW and Application SW), along with the ICU AIV/AIT activities, while the DCUs and TCU requirements specification, design and manufacturing are respectively in charge of France (CEA - Saclay) and Spain (IEEC - Barcelona). These P/L subsystems will be provided to ASI/INAF as external furnished equipments and delivered from CEA and IEEC to ASI/INAF in order to be 
tested, along with the ICU, at INAF premises in Italy, before delivering them to RAL Space (UK) for AIT/AIV activities at Payload level. The AIRS Detector Control Units are implemented in a not redundant configuration and represent the spectrometer warm Front End Electronics located in the SVM, within the ICU box as baseline. They are interfaced to the AIRS CFEE and the T-e2v H1RG detectors for CH0 and CH1 channels (refer to Figure 2) by means of cryoharness in order to control the data generation and acquisition process and constrain the heat load on the FPAs.

The DCU main functions are as follows:

- provide housekeeping for AIRS-OB (optical bench) and AIRS-FPA (detectors and CFEEs) - refer to Figure 3 ;

- control the data acquisition at detector level through the CFEE (from users entry);

- pre-process digital data from the detector (on-board its FPGA);

- control the thermal stabilisation of the detectors through temperature probes and heaters;
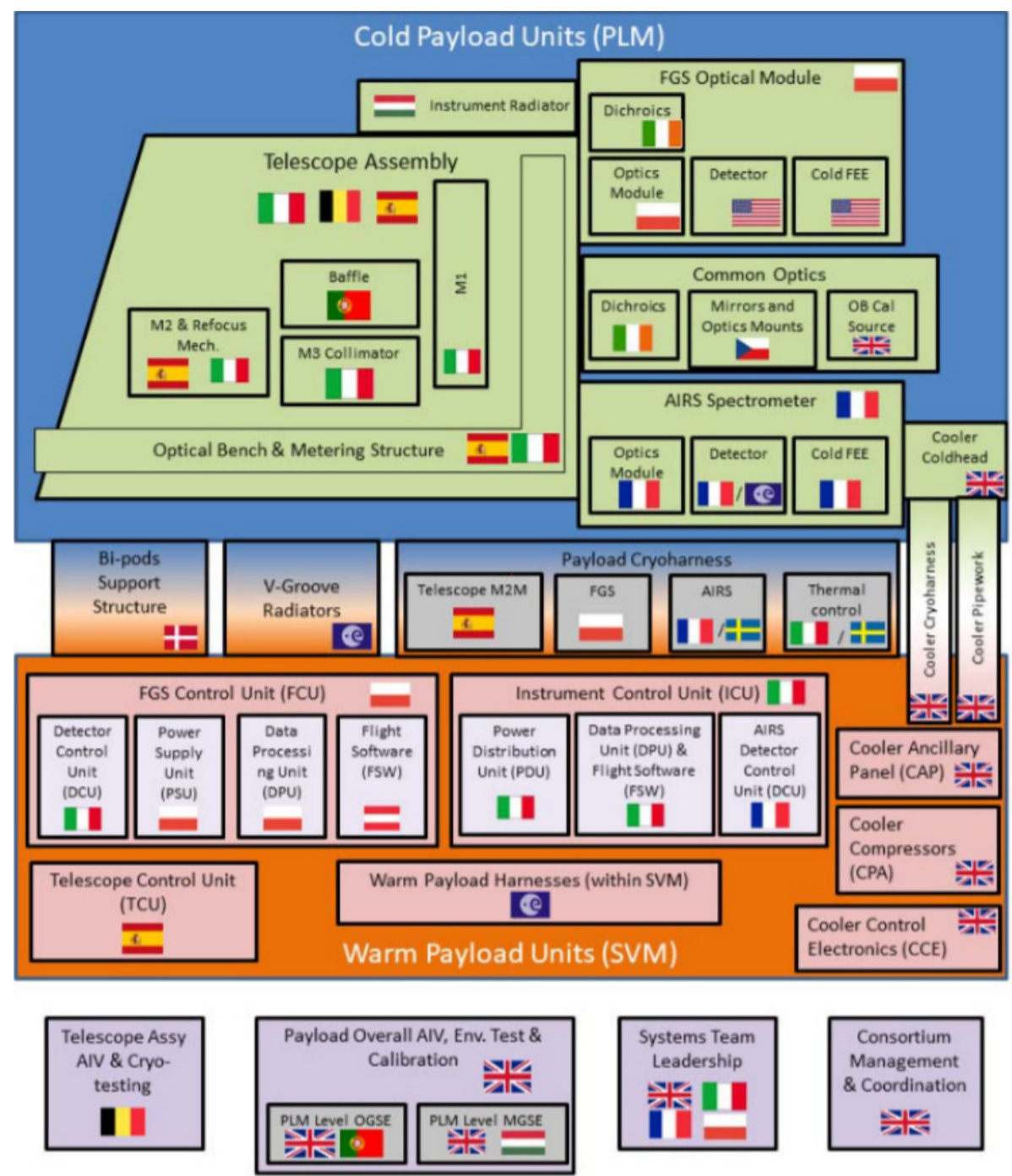

Figure 1. ARIEL Payload block diagram and ARIEL Mission Consortium (AMC) Countries responsibilities - courtesy of the ARIEL Mission Consortium (AMC). 
- interface the ICU CDPU for telecommands (TC) reception, housekeeping (HK) and Science data transmission.

\section{ICU ALLOCATED BUDGETS AND DESIGN DRIVERS}

Table 1 summarises the allocated budgets for the Unit, which need to be considered for the design assessment (the volume allocation for the ICU bending radius is excluded). The ICU science data volume is intended as the nominal weekly allocation sent to the ICU CDPU from AIRS DCUs CH0 and CH1 (equivalent to 317.1 Gbit per week before compression, without margin), excluding internal housekeeping from TCU and DCUs (raw data). Assuming an achievable averaged compression ratio (CR) of 2.5, the expected ICU-to-S/C nominal weekly science plus internal HK data volume is 129.1 Gbit (155.0 Gbit including $20 \%$ of margin).

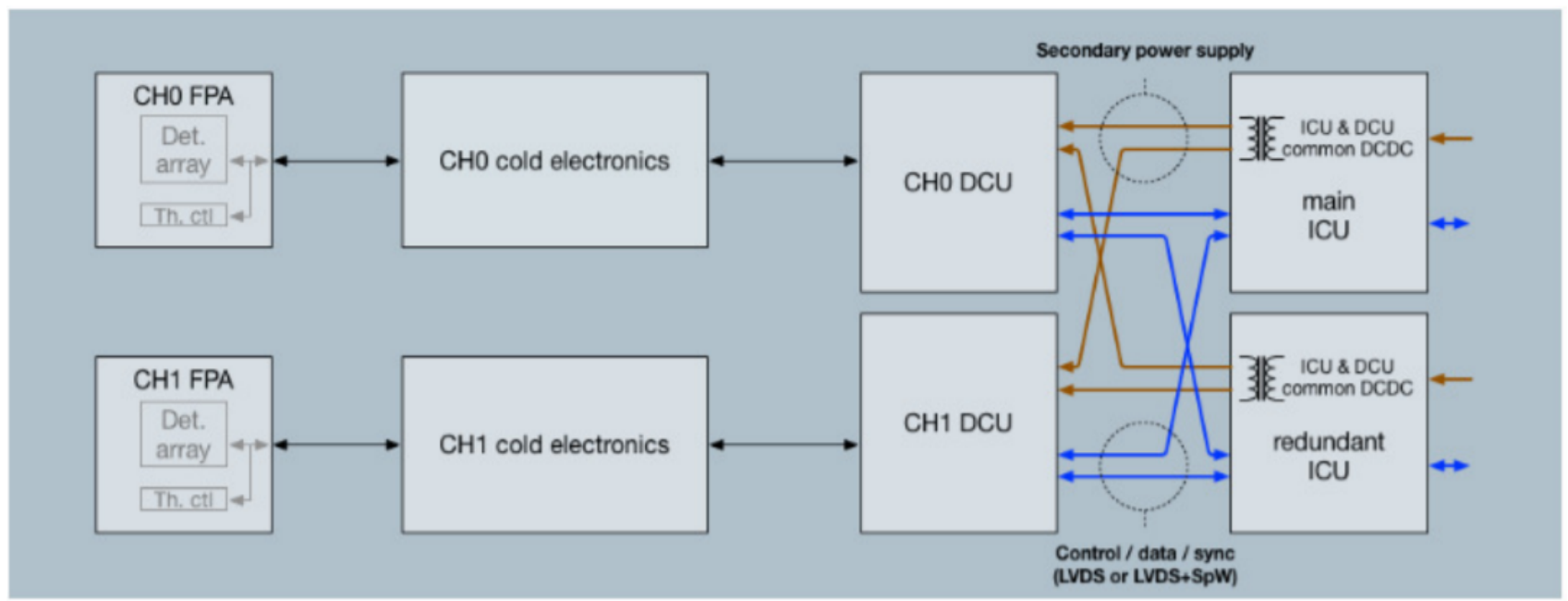

Figure 2. Overview of AIRS electrical system - courtesy of CEA, France
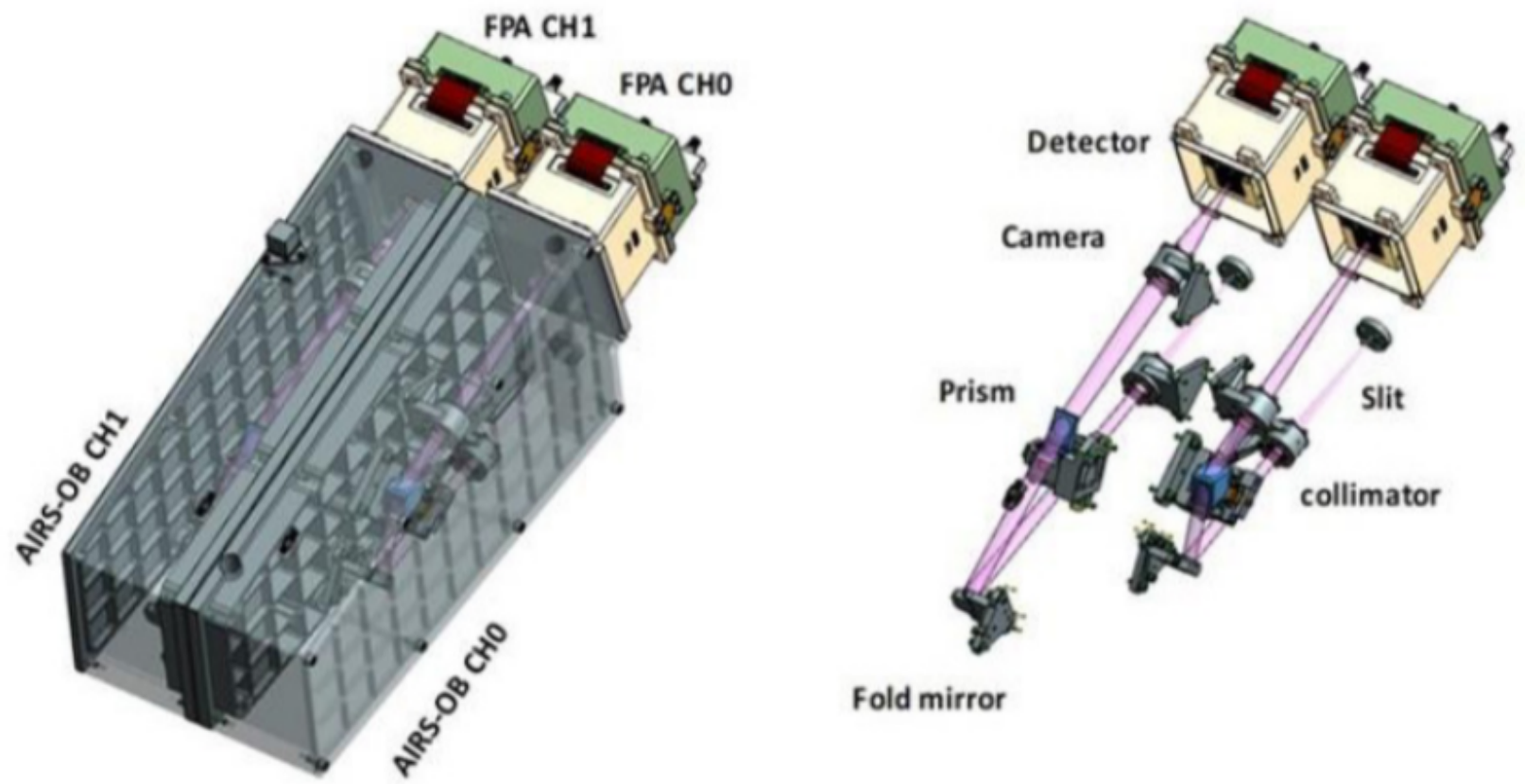

Fold mirror

Figure 3. AIRS Spectrometer showing two joint boxes hosting the optical benches and detectors for $\mathrm{CH} 0$ and $\mathrm{CH} 1 \mathrm{channels}$ - courtesy CEA, France 


\subsection{CDPU input data rate and Housekeeping data rate}

The AIRS input data rate strongly affects the ICU hardware and software design, as the former is managed by the CDPU for the actual needs in terms of buffering and real time data processing. Therefore a basic analysis of the expected input data rate has been performed as an assessment of the overall weekly data volume in terms of HK and science telemetries, in order to correctly size the CDPU on-board memories and the needed processing capabilities. The overall budget of the housekeeping, to be collected by the ICU assembly, is dominated by those related to the TCU subsystem. Anyway, the HK daily volume is negligible compared to the concerning scientific data (see Table 1).

\subsection{Science data rate and data volume assessment criteria}

The detectors selection for the ARIEL Spectrometer is based both on the Teledyne MCT 1k x 1k array already developed for the NASA's NEOCam mission and the heritage of the WISE mission; this kind of detectors allow for non-destructive (or multi-accumulate sampling up-the-ramp) readout modes. This capability can effectively reduce the equivalent readout noise, improving the signal to noise ratio and allowing for example an easier identification and rejection of glitches induced by cosmic rays hits affecting the signal. Data coming from AIRS CH0 and CH1 CFEEs are firstly managed by the two AIRS DCUs and then transferred across the Spacewire (SpW) RMAP (Remote Memory Access Protocol) links to CDPU. Data from detectors/CFEEs are represented by cropped windows of $300 \times 64$ pixels for $\mathrm{CH} 0$ and $100 \times 64$ pixels for $\mathrm{CH} 1$ with a pixel depth of 16 bit (2 bytes). If we assume to sample up-the-ramp pixels in a non-destructive manner with the length of the ramp determined by the saturation limits of the detector, an estimation of the expected data rate can be provided, in theory, for any target of known flux (bright, medium, faint). The performed estimation is including the need of having a detector reset between each ramp or a correlated double sampling (CDS). The ARIEL P/L overall weekly data budget has been calculated and reported in Table 2, where a breakdown of the expected data rate including the housekeeping collected by ICU (TCU included) is provided. The Payload data volume is dominated by the FGS NIRSpec, AIRS-CH0 and AIRS-CH1 channels and takes into account the observing efficiency (95\% - targets plus calibration), as well as the system margin. The calculation provided in Table 2, on which has been defined the assigned budget of Table 1, assumes on-board fitting up the ramp or CDS (Correlated Double Sampling), with an average ramp length to saturation up-to few seconds for the AIRS channels and a following DCU data decimation. In particular, it is assumed that we sample up-the-ramp pixels in a nondestructive manner with a relative high frame rate $(5.8 \mathrm{~Hz}$ for AIRS-CH0,15.8 Hz for AIRS-CH1), followed by destructive readouts after a defined number of samples, depending on the brightness of the target.

The actual read-out mode to be used will actually vary between targets depending on their brightness, with the possibility of setting the ramp integration time in the range of few seconds. For each star target the expected flux will be used for refining the best readout scheme and obtaining the corresponding data rate. The adopted scheduling tool for ARIEL observations, calibrations and data delivery to Ground, will also be used to show how the payload data rate may vary throughout the mission and to evaluate the expected maximum and average data rates, thus allowing for a final dimensional estimation of the on-board processing needs (along with the ICU buffering capabilities), prior to sending such data to the S/C Mass Memory Unit (MMU). As most of the

\begin{tabular}{|l|l|r|l|l|l|l|}
\hline & $\begin{array}{c}\text { Power } \\
(\mathbf{W})\end{array}$ & $\begin{array}{c}\text { Mass } \\
(\mathbf{k g})\end{array}$ & $\begin{array}{c}\text { Dimensions } \\
\mathbf{( m m}) \\
\mathbf{L} \mathbf{~ W} \mathbf{~} \mathbf{H}\end{array}$ & $\begin{array}{c}\text { AIRS weekly } \\
\text { data volume } \\
\text { before } \\
\text { compression } \\
\text { (Gbit) }\end{array}$ & $\begin{array}{c}\text { AIRS weekly } \\
\text { data volume } \\
\text { after } \\
\text { compression } \\
\text { (Gbit) }\end{array}$ & $\begin{array}{c}\text { HK } \\
\text { weekly } \\
\text { volume } \\
\text { (Gbit) }\end{array}$ \\
\hline Allocated & 29.1 & 6.7 & N/A & 317.1 & 126.8 & 2.3 \\
\hline Margin $(20 \%)$ & 5.8 & 1.3 & N/A & 63.3 & 25.4 & 0.5 \\
\hline $\begin{array}{l}\text { Total with } \\
\text { margin }\end{array}$ & $\mathbf{3 4 . 9}$ & $\mathbf{8 . 0}$ & $\mathbf{3 2 0} \times \mathbf{1 8 5 . 1} \times \mathbf{2 2 0}$ & $\mathbf{3 8 0 . 4}$ & $\mathbf{1 5 2 . 2}$ & $\mathbf{2 . 8}$ \\
\hline
\end{tabular}

Table 1. ICU allocated budgets, DCUs included 


\begin{tabular}{|l|l|r|}
\hline \multicolumn{1}{|c|}{$\begin{array}{c}\text { ARIEL } \\
\text { subsystem }\end{array}$} & \multicolumn{1}{|c|}{ Description } & $\begin{array}{c}\text { Requirement } \\
\text { as output } \\
\text { data rate to } \\
\text { SVM } \\
\text { (Gbit/week) }\end{array}$ \\
\hline FGS & Science data + AOCS data stream + internal HK & 64.2 \\
\hline ICU Science & Science data + internal HK & 126.8 \\
\hline ICU/TCU PLM HK & Thermal monitoring + M2M TM + Cal Unit + others & 2.3 \\
\hline CCE HK & Cooler HK \& TM & 0.8 \\
\hline TOTAL & \multicolumn{1}{|c|}{} & $\mathbf{1 9 4 . 1}$ \\
\hline & System Margin $(20 \%)$ & 41.9 \\
\hline Margin & TOTAL ARIEL PLM allocation & $\mathbf{2 3 6}$ \\
\hline $\begin{array}{l}\text { TOTAL with } \\
\text { margin }\end{array}$ & Allocation pre-compression from AIRS DCUs to ICU CDPU & $\mathbf{3 1 7 . 1}$ \\
\hline
\end{tabular}

Table 2. Overall ARIEL Payload data rate budget. In red is highlighted the expected data flow from AIRS, prior ICU on-board compression

expected data processing (e.g. compression) will be performed in quasi real time, no particular criticality for data buffering is presently foreseen.

\section{ICU ELECTRICAL DESIGN}

The AIRS-ICU schematic diagram is represented in Figure 4 where the single boards (nominal and redundant, $\mathrm{CHO}$ and $\mathrm{CH} 1$ ) and the internal and external electrical interfaces are shown. The final design will discriminate between two possible alternatives regarding the Thermal Control stabilisation System (TCS) design and implementation. The choice is between:

- Adoption of a DCU design from CEA (baseline design), implementing an embedded TCS for AIRS;

- Adoption of a DCU design from INAF (alternative design), exploiting the NISP DCU design from OHBItaly for the Euclid Mission, but implementing the AIRS TCS on the ICU Power Supply Unit (PSU).

In both cases the interfaces between ICU and SVM do not change; while minor changes will concern ICU internal and ICU to AIRS CFFE connections. The baseline design foresees that the AIRS TCS system is implemented on board the AIRS DCU, instead, in Figure 4 it is shown the second design with the AIRS Thermal Control Stabilisation inside the PSU board.

The PSU board has enough free space to host the TCS in case the alternative design should be chosen. The ICU architecture is based on a partial cold-redundant and cross-strapped configuration, as shown in Figure 4 and this is independent on the DCU adopted design. Both DCU are cross-strapped and can work indifferently with the nominal (black arrows) or redundant (red arrows) PSU and CDPU; anyway, DCU themselves are not redundant. This design is inherited from the NISP Instrument ${ }^{14}$ on board the Euclid Mission. In the NISP case it has been assessed an overall reliability greater than $98 \%$ (and a Technology Readiness Level of 8) and for this reason it was decided, for the ARIEL mission, to avoid the increasing in complexity, power, mass and volume created by the implementatibcn of two redundant DCU.

The benefits of adopting the DCU-SIDECAR alternative design consist in the aforementioned high TRL deriving from the 16 DCU Flight Models already manufactured and fully tested, but also on the possible reuse for both AIRS and FGS of the same DCU-SIDECAR Interface simulator, an approach already adopted by the NISP Team. 
In the following Figure 5 are reported the ICU Nominal and Redundant electrical interfaces towards the SVM (in orange), towards AIRS (in green, for the alternative design) and to the TCU (in red). Also the Debug Support Unit interface for CDPU is shown (in white). No cross-strapping is presently foreseen between the SVM warm units in charge of the S/C Prime (On-Board Computer and MMU) and ICU. As anticipated, the electrical interfaces to the SVM and TCU are valid for both the baseline and the alternative ICU designs.

\section{ICU ON-BOARD SOFTWARE AND DATA HANDLING FUNCTIONALITIES}

In the following, a summary of all the ICU data handling functionalities is provided, with an indication of the involved unit:

- CDPU: TC handling, Instrument (AIRS) commanding and control, science data acquisition and compression (lossless algorithm, SW or mixed HW/SW implementation), ICU and P/L HK acquisition and monitoring, instrument FDIR implementation, data packetisation and TM flow management;

- DCU: AIRS detectors and CFEEs (CH0 andCH1) management, data flow control by means of algorithms based on a HW implementation (FPGA-based);

- PSU: no data handling functions are implemented on-board, HW HK data only (ICU internal currents, voltages and temperatures) will be collected and converted in digital format, to be sent to CDPU for active monitoring.

In particular, the Ariel On Board Software (OBSW) running on the CDPU board will be composed of the following three main components:

- Boot Software: it is installed on the PROMs of the ICU CDPU board and allows loading/deploying the ICU Application Software in RAM. It comprises all the low level drivers for the CDPU board and its related interfaces involved in the bootstrap process.

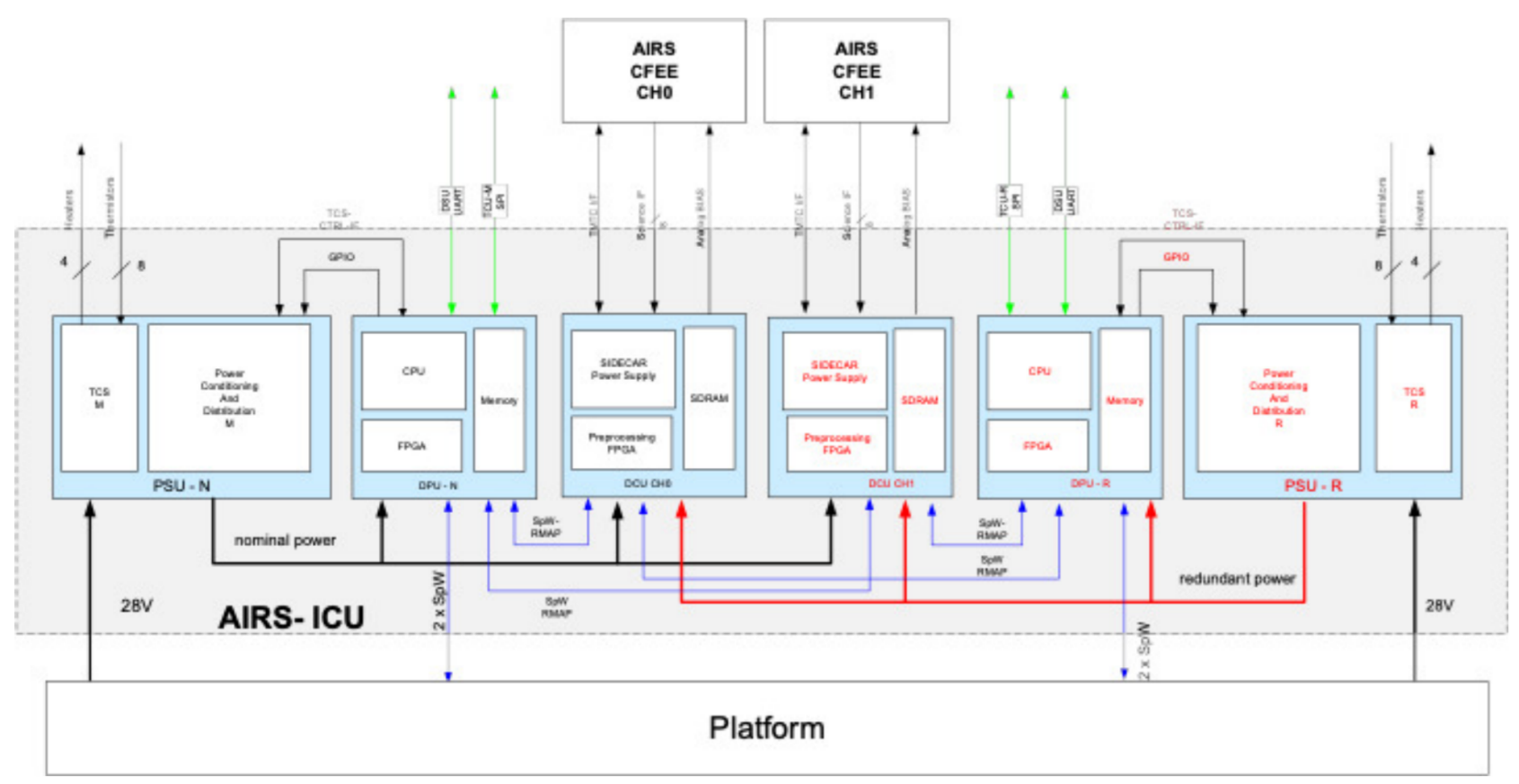

Figure 4. ARIEL ICU block diagram showing the electrical interfaces towards the SVM (Platform) and P/L (Payload) 
- Basic Software, or Basic Support Package (BSP): basic I/O SW, Service SW \& Peripheral Drivers; it is a hardware-dependent Software including the Software Drivers for all the internal and external ICU digital interfaces. This SW is used by the Application SW and can depend on the selected operating system (OS).

- Application Software: it is composed by two parts:

- the Instrument Control \& Configuration Software: it implements the ARIEL scientific payload handling, TCU included. It controls the AIRS spectrometer, implements the operating modes, monitors the instrument health and runs FDIR procedures. It implements the interface layer between the S/C and the instrument.

- the AIRS Data Processing and Compression Software ${ }^{15}$ : it implements all the necessary on-board processing functionalities, included the on-board lossless compression. After the compression the SW prepares CCSDS packets for the transmission to the S/C Mass Memory Unit (MMU).

Boot SW and HW-dependent SW are strictly related to the ICU HW. Their development will be part of the activities included in the industrial contract for the ICU provision. The criticality analysis for these SW components will be part of the documentation to be provided by the industry, which will be selected as ICU Prime Contractor. For this part of SW components the ICU Prime shall also consider the ESA-SAVOIR (Space AVionics Open Interface aRchitecture) guidelines and alternatives. The Real time operating system will be purchased as a commercial off-the-shelf (COTS) part.

\section{ICU THERMO-MECHANICAL DESIGN AND ANALYSES}

\subsection{Mechanical design}

The ICU 3D mechanical design is shown in Figure 6. It foresees a set of six drawers (refer to Figure 7) plugged into a back-plane supported by the bottom panel, hosting the connectors for the following PCBs:

\begin{tabular}{|c|c|c|}
\hline Electrical I/F & Type & Remarks \\
\hline Primary power input $\mathbf{N}$ & $28 \mathrm{~V}$ not regulated & S/C PCDU link \\
\hline Primary power input $\mathbf{R}$ & $28 \mathrm{~V}$ not regulated & S/C PCDU link \\
\hline TMTC SDW N & $\mathrm{SpW}$ main link for TM/TC with the $\mathrm{S} / \mathrm{C}$ & $\mathrm{S} / \mathrm{C}$ OBC link \\
\hline TM SpWW N & SpW main link for science data & S/C SSMM link \\
\hline TMTC SDW R & SpWW redu link for TM/TC with the S/C & $\mathrm{S} / \mathrm{C}$ OBC link \\
\hline TM SpW R & SpW redu link for science data & S/C SSMM link \\
\hline ICU N SPI & SPI link with TCU-M & $P / L$ internal link \\
\hline CORSPI & SPI link with TCU-R & $P / L$ internal link \\
\hline DSU UART N & RS422 & Debug I/F \\
\hline DSU UART R & RS422 & Debug I/F \\
\hline SIDECAR I/F Ch0 & $\begin{array}{l}\text { TMTC serial I/F - LVDS } \\
8 \text { bit parallel science I/F -LVDS } \\
\text { Various analog bias }\end{array}$ & $\begin{array}{l}\text { P/L internal link } \\
\text { (partial cryo- } \\
\text { harness from PIP } \\
\text { to CFEE) }\end{array}$ \\
\hline SIDECAR I/F Ch1 & $\begin{array}{l}\text { TMTC serial I/F - LVDS } \\
8 \text { bit parallel science I/F -LVDS } \\
\text { Various analog bias }\end{array}$ & $\begin{array}{l}\text { P/L internal link } \\
\text { (partial cryo- } \\
\text { harness from PIP } \\
\text { to CFEE) }\end{array}$ \\
\hline TCS I/F N & $\begin{array}{l}4 \text { heaters } \\
8 \text { thermistors }\end{array}$ & $\begin{array}{l}\text { P/L internal link } \\
\text { (partial cryo- } \\
\text { harness from PIP } \\
\text { to CFEE) }\end{array}$ \\
\hline TCS I/F R & $\begin{array}{l}4 \text { heaters } \\
8 \text { thermistors }\end{array}$ & $\begin{array}{l}\text { P/L internal link } \\
\text { (partial cryo- } \\
\text { harness from PIP } \\
\text { to CFEE) }\end{array}$ \\
\hline
\end{tabular}

Figure 5. ICU alternative design electrical I/F 
- 2x PSU (N \& R) boards

- 2x CDPU (N \& R) boards

- 2x DCU (both N) boards

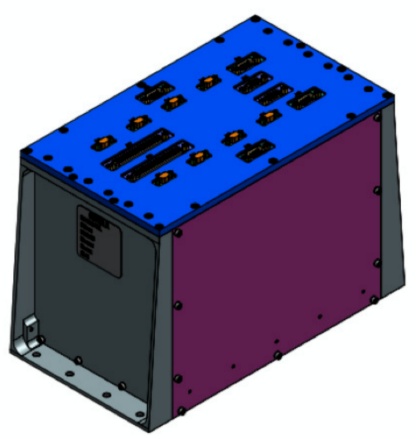

Figure 6. ICU box mechanical design.

The present allocated dimensions by ESA for the box envelope are $320 \mathrm{~mm} \times 185.1 \mathrm{~mm} \times 220 \mathrm{~mm}$ (length $\times$ width $\times$ height). Presently, the ICU dimensions from CAD design are $292.5 \mathrm{~mm} \times 158.6 \mathrm{~mm} \times 197.6 \mathrm{~mm}$, so some margin (more than $10 \mathrm{~mm} /$ dimension) is still available for a better accommodation of PCB, EEE components and tracks routing during the PCBs executive design phase. The present dimensions for the ICU box design concern the implementation of the NISP version of the DCU design, while for the CEA customised design implementation an increase in height is expected up to $270 \mathrm{~mm}$ in order to include the AIRS TCS on-board DCU. The definition of the electronic boards dimensions and box height is still under assessment and shall consider the possibility to adopt a piggy-board configuration (refer to Figure 8). The panels of the ICU box will be manufactured in an Aluminium alloy and then externally painted in black (except the bottom panel) if needed to improve radiating exchange with the environment, while assuring a proper thermal conduction towards the SVM bench. The ICU box will internally host the grounding reference point along with a bounding stud and at least a $\mathrm{N}$ and a $\mathrm{R}$ TRP (Temperature Reference Point) for monitoring the Unit temperature (in charge of S/C). The drawers thermomechanical coupling with the box structure will be done thanks to the adoption of card-lock retainers, ensuring a proper thermal conduction and heat dissipation toward the SVM bench by means of the lateral panels and ribs. Connectors shown in the mechanical design are not fully representative, but it is expected to adopt MDM micro-d type, 9 poles, for SpW TM/TC and SPI signals; DSUB, 9 poles, for power signals).

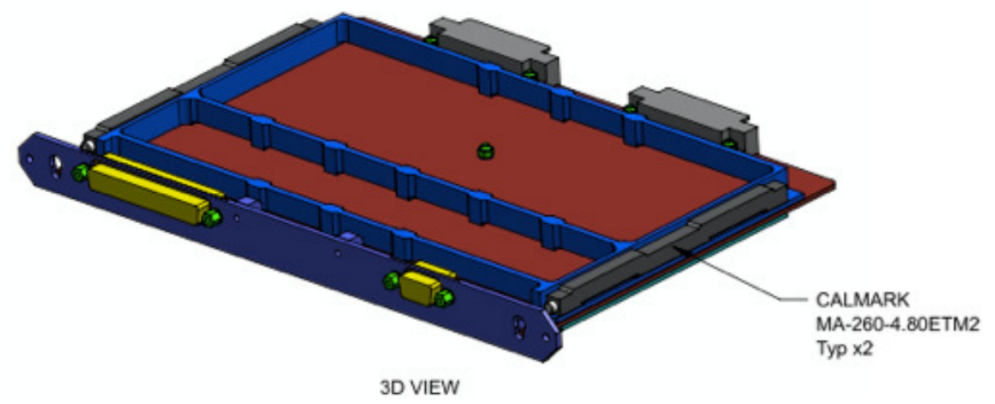

Figure 7. 3D view of a PCB with its mechanical frame and card-lock retainers for stiffness improvement and a proper thermal dissipation to the box and to SVM bench.

\subsection{Structural model and analyses}

The ICU structural analysis has been carried out with a NASTRAN model, whose expected FEM mass is $6.7 \mathrm{~kg}$ ( $20 \%$ of mass contingency excluded). The main result of the structural analysis, the 1st eigenfrequency equal to 


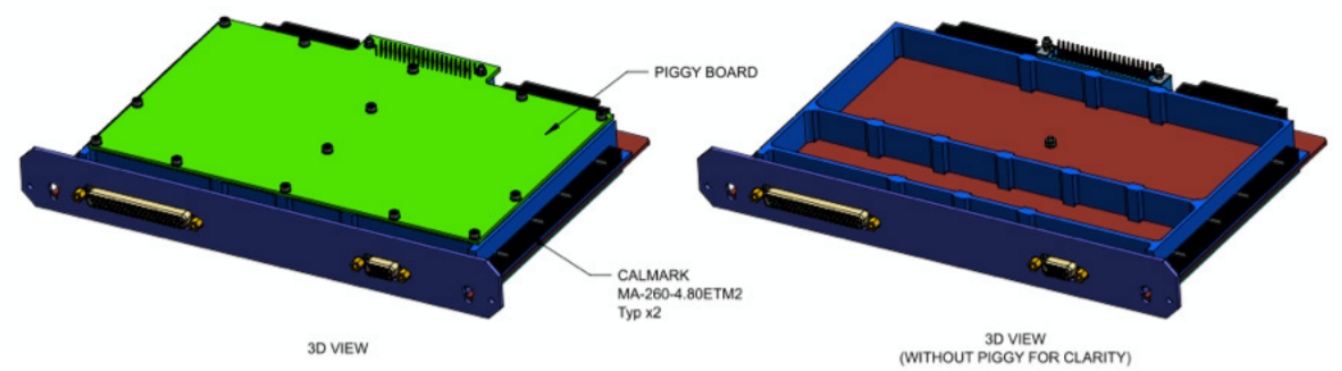

Figure 8. 3D view of a customised PCB hosting a piggy-board.

189.77 Hz, is larger than the required value of $140 \mathrm{~Hz}$, as shown in Figure 9. DLL (Design Limit Loads) stresses have then been applied to the unit (20g inplane, $25 \mathrm{~g}$ out-of-plane) and resulting stresses have been extracted, showing that all Margins of Safety (MoS) are positive (refer to Table 3).

\begin{tabular}{|cccccccc|}
\hline MODE N. FREQ. [HZ] & $\begin{array}{c}\text { EFF. } \\
\text { MASS TX }\end{array}$ & $\begin{array}{c}\text { EFF. } \\
\text { MASS TY }\end{array}$ & $\begin{array}{c}\text { EFF. } \\
\text { MASS Tz }\end{array}$ & $\begin{array}{c}\text { EFF. } \\
\text { MASS RX }\end{array}$ & $\begin{array}{c}\text { EFF. } \\
\text { MASS RY }\end{array}$ & $\begin{array}{c}\text { EFF. } \\
\text { MASS Rz }\end{array}$ \\
\hline $\mathbf{1}$ & 189.77 & $\mathbf{6 7 . 1 4} \%$ & $0.00 \%$ & $0.02 \%$ & $0.01 \%$ & $\mathbf{6 6 . 8 2} \%$ & $\mathbf{3 0 . 6 0} \%$ \\
\hline $\mathbf{2}$ & 261.25 & $0.00 \%$ & $0.00 \%$ & $1.00 \%$ & $0.41 \%$ & $0.29 \%$ & $0.00 \%$ \\
\hline $\mathbf{3}$ & 274.45 & $0.03 \%$ & $0.00 \%$ & $0.00 \%$ & $0.00 \%$ & $0.27 \%$ & $0.01 \%$ \\
\hline $\mathbf{5}$ & 292.11 & $0.00 \%$ & $0.00 \%$ & $0.00 \%$ & $0.00 \%$ & $0.05 \%$ & $0.00 \%$ \\
\hline $\mathbf{9}$ & 301.46 & $0.00 \%$ & $0.00 \%$ & $0.00 \%$ & $0.00 \%$ & $0.00 \%$ & $0.00 \%$ \\
\hline $\mathbf{1 1}$ & 309.36 & $0.00 \%$ & $0.00 \%$ & $\mathbf{5 . 5 7} \%$ & $2.29 \%$ & $1.64 \%$ & $0.00 \%$ \\
\hline $\mathbf{1 2}$ & 474.08 & $0.00 \%$ & $\mathbf{5 3 . 5 3} \%$ & $0.00 \%$ & $\mathbf{5 8 . 6 7} \%$ & $0.00 \%$ & $\mathbf{1 2 . 5 9} \%$ \\
\hline $\mathbf{4 7}$ & 761.21 & $0.00 \%$ & $\mathbf{3 8 . 0 6} \%$ & $0.00 \%$ & $0.25 \%$ & $0.00 \%$ & $\mathbf{9 . 2 6} \%$ \\
\hline $\mathbf{1 0 5}$ & 1122.84 & $0.00 \%$ & $0.00 \%$ & $0.00 \%$ & $0.00 \%$ & $0.00 \%$ & $\mathbf{7 . 7 7} \%$ \\
\hline
\end{tabular}

Figure 9. ICU eigenfrequencies

\begin{tabular}{|c|c|c|c|c|c|c|c|c|}
\hline ITEM & $\begin{array}{c}\text { LOAD } \\
\text { CASE }\end{array}$ & $\begin{array}{c}\text { Fty } \\
{[\mathbf{M P a}]}\end{array}$ & $\begin{array}{c}\text { Ftu } \\
{[\mathbf{M P a}]}\end{array}$ & $\begin{array}{c}\text { Limit } \\
\text { Stress } \\
{[\mathbf{M P a}]}\end{array}$ & $\mathbf{S F}_{-} \gamma$ & $\mathbf{S f}_{-} \mathbf{U}$ & $\mathbf{M o S}_{-} \gamma$ & $\mathbf{M o S} \mathbf{U}$ \\
\hline Aluminium parts & 101 & 386 & 462 & 48.1 & 1.10 & 1.25 & 6.30 & 6.68 \\
\hline PCB & 101 & NA & 482 & 5.99 & NA & 1.25 & NA & 63.37 \\
\hline
\end{tabular}

\subsection{Thermal model and analyses}

The ICU thermal analysis has been firstly developed considering only the conductive processes through the baseplate during the heat exchange with the environment. Both the conductive and the radiative processes have been taken into account for the internal heat exchange with the following values:

- Mechanical elements: Al7075 k $=130 \mathrm{~W} / \mathrm{mK}, \epsilon=0.03$

- PCB: Copper $\mathrm{k}=400 \mathrm{~W} / \mathrm{mK}$ (equivalent thickness), $\epsilon=0.8$

and a qualification temperature of $50{ }^{\circ} \mathrm{C}$ at TRP (Temperature Reference Point). EEE components heat dissipation has been considered evenly distributed on PCB modules as reported in Table 4, showing the expected 


\begin{tabular}{|l|l|}
\hline \multicolumn{1}{|c|}{ Board } & \multicolumn{1}{c|}{$\begin{array}{c}\text { Heat load [W] } \\
\text { (20\% contingency included })\end{array}$} \\
\hline PSU Main & 14.4 \\
\hline CDPU Main & 9.42 \\
\hline DCU CH0 & 3.96 \\
\hline DCU CH1 & 3.96 \\
\hline CDPU Red & OFF \\
\hline PSU Red & OFF \\
\hline Total & $\mathbf{3 1 . 7 4}$ \\
\hline
\end{tabular}

heat loads on each electronic board (Nominal, Redundant and cross-strapped). The thermal analysis has shown the following results (predicted temperatures):

- Max calculated Tcalc $=69.4{ }^{\circ} \mathrm{C}$ on active PSU PCB

- Max predicted Tpred $=$ Tcalc + uncertainty $=69.4+10=79.4{ }^{\circ} \mathrm{C}$

showing no issues for the operability of the selected main EEE components.

\section{CONCLUSIONS}

With this paper, the role of the Instrument Control Unit within the ARIEL Payload along with its updated electrical and thermo-mechanical design, following the ESA's P/L System Requirements Review (pSRR), have been presented. Some design options are still open, in particular concerning the DCU architecture driving the AIRS cold front-end electronics (SIDECAR ASIC or based on discrete EEE components) that, regardless of its final implementation, requires particular care for the components selection, its thermal stability and the capability to provide noise-free and very stable voltage levels towards the CFEEs. These thermo-electrical stability requirements shall be further assessed after the Mission Adoption Review (MAR), ended in November 2020, once better known the ARIEL Mission operational scenario (e.g. S/C pointing, slews, etc.) and the ICU thermo-mechanical environment and constraints, as defined thanks to the next co-engineering sessions with ESA and the Spacecraft Prime, whose selection and negotiation phase is expected in summer of the next year, following Invitation To Tenders (ITT) processes.

\section{ACKNOWLEDGMENTS}

The authors would like to thank the UK Science and Technology Facilities Council (STFC), the Italian Space Agency (ASI) for the financial contribution to the ARIEL project in the framework of the ASI/INAF agreement 2018.22.HH.0 (Accordo Attuativo della Convenzione Quadro), the French CNES (Centre National d'Etudes Spatiales) and OHB Italy for the support to the preliminary design of the ICU and DCU boards, for both AIRS and FGS instruments on-board ARIEL. Finally, a special thank to the Ariel Study Team of the European Space Agency, for the invaluable support provided to the ARIEL Mission Consortium.

\section{REFERENCES}

[1] Tinetti, G., "ESA M4 Ariel: Phase B." EPSC-DPS Joint Meeting (2019).

[2] Puig, L. et al., "The phase a study of the esa m4 mission candidate ariel," Experimental Astronomy XX, XX (2018).

[3] Pascale, E. et al., "The Ariel space mission," in [International Conference on Space Optics - ICSO 2018], Proc. SPIE 10698(106980H) (2018).

[4] Middleton, K. et al., "An integrated payload design for the atmospheric remote-sensing infrared exoplanet large-survey (Ariel): results from phase A and forward look to phase B1," in [International Conference on Space Optics - ICSO 2018], Proc. SPIE 11180(1118036) (2019). 
[5] Eccleston, P. and Tinetti, G., "A summary of the payload design for Ariel." European Planetary Science Congress (2018).

[6] Eccleston, P. et al., "The ariel payload," Experimental Astronomy Special Issue on Ariel, XX (2017).

[7] Tinetti, G. et al., "A chemical survey of exoplanets with ariel," Experimental Astronomy XX, XX (2018).

[8] Tinetti, G. et al., "The ariel science case," Experimental Astronomy Special Issue on Ariel, XX (2017).

[9] Amiaux, J. et al., "Airs, the ariel spectrometer," Experimental Astronomy Special Issue on Ariel, XX (2017).

[10] Focardi, M. et al., "The ariel instrument control unit design for the m4 mission selection review of the esa's cosmic vision program, experimental astronomy," Experimental Astronomy XX, XX (2018).

[11] Rataj, M. et al., "The fine guidance system (fgs) of the ariel payload," Experimental Astronomy Special Issue on Ariel, XX (2017).

[12] Sierra-Roig, C. et al., "The ariel esa mission on-board metrology," in [Proceedings of the 4th IEEE International Workshop on Metrology for Aerospace], XX(XX) (2017).

[13] Crook, M. et al., "Development of a 2k joule-thomson closed-cycle cryocooler," in [International Cryocooler Conference, Inc., Boulder (CO)], XX(XX) (2016).

[14] Corcione, L. et al., "The data processing unit of the nisp instrument of the euclid mission," in $[X X]$, Proc. SPIE 9143(914331) (2014).

[15] et al, M. F., "Ariel spectrometer instrument control and data processing software," European Planetary Science Congress (EPSC) (2017). 